\title{
Hunting for a scalar glueball in exclusive B decays
}

\author{
Cai-Dian L $\ddot{u}^{1}$, Ulf-G. Meißner ${ }^{2,3}$, Wei Wang ${ }^{2, a}$, and Qiang Zhao ${ }^{1}$ \\ 1 Institute of High Energy Physics and Theoretical Physics Center for Science Facilities, Chinese Academy of Sciences, Beijing \\ 100049, China \\ 2 Helmholtz-Institut für Strahlen- und Kernphysik and Bethe Center for Theoretical Physics, Universität Bonn, D-53115 Bonn, \\ Germany \\ 3 Institute for Advanced Simulation, Institut für Kernphysik and Jülich Center for Hadron Physics, JARA-FAME and \\ JARA-HPC, Forschungszentrum Jülich, D-52425 Jülich, Germany
}

Received: 29 March 2013 / Revised: 3 May 2013

Published online: 21 May 2013

(C) The Author(s) 2013. This article is published with open access at Springerlink.com

Communicated by M. Anselmino

\begin{abstract}
Although glueballs, as one of the type of exotic hadrons allowed by QCD, have been well established on the lattice, experimental searches up to this date for bound states of gluons have only produced controversial signals. In this work, using flavor SU(3) symmetry for the light quarks validated by the available experimental data, we propose an intuitive way to hunt for a scalar glueball in exclusive $B$ decays. In the presence of mixing between the glueball and ordinary scalar mesons, we point out that the scalar glueball content amounts to the ratio of B-decay branching fractions. We discuss the implication from the recently available experimental data and show the sensitivities of $B$ decays as a probe to the scalar structures. The on-going LHCb experiment and forthcoming Super KEKB factory would allow access to precisely establishing the mixing pattern among the scalars, and may allow one to disentangle the long-standing puzzle concerning the existence and mixings of the scalar glueball predicted by QCD.
\end{abstract}

\section{Introduction}

QCD allows the existence of hadronic states beyond the quark model description of mesons and baryons. Among these exotic states, glueballs are bound states made of the color force carriers only. Lattice QCD simulations have suggested the mass of the lowest-lying scalar glueball around $1.5-1.8 \mathrm{GeV}[1-9]$. Despite several possible candidates in this mass region, the existence of a scalar glueball state is still under debate, largely because of the fact that the lowest-lying scalar glueball has the same quantum numbers as the QCD vacuum, and thereby mixes with ordinary quark-antiquark states.

Most glueball studies available in the literature have focused on decay properties and the production in lowenergy processes. In fact, the study of the production in $B$ decays is another powerful way to uncover the mysterious structure of scalar mesons and figure out the gluon component inside [10-15].

The motivation of this work is to provide an up-todate analysis of $B$ decays into a scalar meson plus a $J / \psi$, particularly in the light of the recent data on $B / B_{s} \rightarrow$ $J / \psi \pi^{+} \pi^{-} / K^{+} K^{-}$decays from the LHCb, Belle and D0 Collaborations [16-20]. In view of these, we will suggest an intuitive way for the identification of a glueball.

\footnotetext{
a e-mail: weiwang@hiskp.uni-bonn.de
}

In the following, we shall consider three scalar mesons $f_{0}(1370), f_{0}(1500)$ and $f_{0}(1710)$ all having potentially a large glue content, see, for instance, refs. [21-23]. These three mesons, together with the isotriplet $a_{0}(1450)$ and isodoublet $K_{0}^{*}(1430)$ can form an $\mathrm{SU}(3)$ nonet made of $\bar{q} q$, with one additional state arising from the mixing with the glueball [24]. From this viewpoint, the isosinglet scalar meson among $f_{0}(1370), f_{0}(1500)$ and $f_{0}(1710)$ is expanded as

$$
\left|f_{0}\right\rangle=\alpha_{1}|G\rangle+\alpha_{2}|\bar{s} s\rangle+\alpha_{3}|\bar{n} n\rangle
$$

in which the coefficient $\alpha_{1}$ is the measure of the glue content. Here, $n$ denotes the light quark flavors up and down and we work in the isospin limit in what follows. When other Fock states are included, it is likely that $\alpha_{1}^{2}+\alpha_{2}^{2}+$ $\alpha_{3}^{2}<1$ due to the unitarity condition. However since most of the available Lattice QCD simulations suggest that the mass of the scalar glueball is in the range from $1.5 \mathrm{GeV}$ to $1.8 \mathrm{GeV}$, it is reasonable to believe that glueball will dominate in one of the mesons discussed here. In this case, it is plausible to neglect the subdominant components, leading to $\alpha_{1}^{2}+\alpha_{2}^{2}+\alpha_{3}^{2} \simeq 1$. Other alternative mixing schemes of scalar mesons are also proposed in the literature, and, for instance, ref. [25] has discussed the route in which the scalar glueball may mix with the $f_{0}(980)$. However our following discussion is still valid as long as the glue content dominates in one of the scalar mesons. 


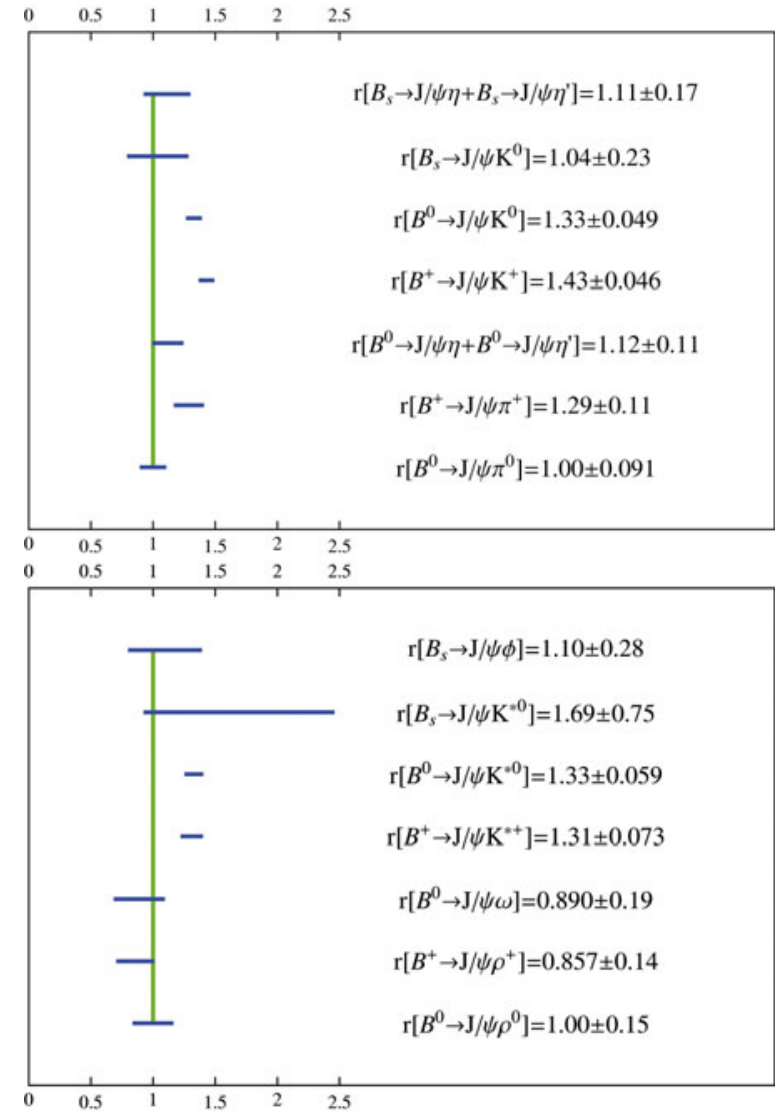

Fig. 1. Ratios of branching fractions of $B \rightarrow J / \psi P$ and $B \rightarrow$ $J / \psi V$. The vertical lines denotes unity and correspond to the $\mathrm{SU}(3)$ symmetry limit.

\section{General analysis based on SU(3) symmetry}

To start, we will assume flavor $\mathrm{SU}(3)$ symmetry for the light $u, d, s$ quarks in the $B \rightarrow J / \psi M$ decay amplitudes. This symmetry has been partly tested in a few $B \rightarrow J / \psi P$ and $B \rightarrow J / \psi V$ processes $[26,27]$ and a good agreement with the data is found. The underlying nature of these processes at the quark level is governed by the $b \rightarrow \bar{c} c s$ or $b \rightarrow \bar{c} c d$ transitions whose matrix elements can be related using the flavor symmetry. For a better comparison to be made in the following, we define the ratio

$$
\begin{aligned}
r\left[B_{q} \rightarrow J / \psi P_{q \bar{q}^{\prime}}\right]= & \frac{\left|V_{c d}\right|^{2}}{\left|V_{c q^{\prime}}\right|^{2}} \frac{\left|C_{\pi^{0}}\right|^{2}}{\left|C_{P}\right|^{2}} \frac{\tau\left(B^{0}\right)}{\tau\left(B_{q}\right)} \\
& \times \frac{\mathcal{B}\left(B_{q} \rightarrow J / \psi P_{q \bar{q}^{\prime}}\right)}{\mathcal{B}\left(B^{0} \rightarrow J / \psi \pi^{0}\right)},
\end{aligned}
$$

whose deviation from unity directly arises from the $\mathrm{SU}(3)$ symmetry breaking effects. In this ratio, $C_{P}=1$ (except for $-C_{\pi^{0}}=C_{\eta_{q \bar{q}}}=1 / \sqrt{2}$ ) is the flavor wave function factor. The ratios for the $B \rightarrow J / \psi V$ processes are defined in a similar way. Using the relevant experimental data for the branching fractions of the various channels [28-30], we collect the results for these ratios in fig. 1 . The vertical lines in this figure denote unity and thus corresponds to the exact SU(3) symmetry limit.
Three observations can be made from the results presented in fig. 1. Firstly, the current uncertainties in the $B_{s}$ decays are large but may get significantly reduced due to the large amount of data accumulated from the LHC and future Super B factories. Secondly, the SU(3) symmetry holds well in the $b \rightarrow d$ processes (namely $B \rightarrow\left(J / \psi \pi, J / \psi \eta^{\left({ }^{\prime}\right)}\right)$ and $\left.B_{s} \rightarrow J / \psi K\right)$, and as well as in the $b \rightarrow s$ transition $\left(B \rightarrow J / \psi K\right.$ and $\left.B_{s} \rightarrow J / \psi \eta^{\left({ }^{\prime}\right)}\right)$. Last, the excess of the branching ratios for the $b \rightarrow s$ processes, roughly $30 \%$, is the same in both $B \rightarrow J / \psi P$ and $B \rightarrow J / \psi V$ decays.

After validating the flavor $\mathrm{SU}(3)$ symmetry, we now explore the consequences in the application to $B \rightarrow J / \psi S$ decays. Suppose in the near future we are equipped with the following experimental data:

$$
\begin{aligned}
& \mathcal{B}_{1}=\mathcal{B}\left(B^{0} \rightarrow J / \psi K_{0}^{*}(1430)\right), \\
& \mathcal{B}_{2}=\mathcal{B}\left(B_{s}^{0} \rightarrow J / \psi K_{0}^{*}(1430)\right), \\
& \mathcal{B}_{3}=\mathcal{B}\left(B_{s}^{0} \rightarrow J / \psi f_{0}\right), \\
& \mathcal{B}_{4}=\mathcal{B}\left(B^{0} \rightarrow J / \psi f_{0}\right),
\end{aligned}
$$

where the second quantity can also be replaced by $\mathcal{B}\left(B^{-} \rightarrow J / \psi a_{0}^{-}(1450)\right)$. The first and third processes are induced by the $b \rightarrow s$ transition, while the other two arise from the $b \rightarrow d$ transition. In the $\mathrm{SU}(3)$ symmetry (to be more specific the U-spin symmetry which interchanges the $s$ and $d$ quarks) limit, $\mathcal{B}_{1}=\mathcal{B}_{2}$, but in order to account for the symmetry breaking effects that can reach $30 \%$ as we have shown, we will retain the differences in $\mathcal{B}_{1}$ and $\mathcal{B}_{2}$. This treatment will refine our analysis based on the $\mathrm{SU}(3)$ symmetry and greatly reduce the systematic errors in the analysis. Using these four branching ratios, we also introduce the quantity

$$
\mathcal{R}=1-\mathcal{B}_{3} / \mathcal{B}_{1}-2 \mathcal{B}_{4} / \mathcal{B}_{2}
$$

In the leading-Fock-state approximation, a scalar glueball is composed of two constituent gluons. In exclusive $B$ decays, the two gluons can be emitted from either the heavy $b$ quark or the light quark. The emission of a collinear gluon from the heavy $b$ quark is suppressed by $1 / m_{b}^{2}$. Since the initial state does not contain any valence gluon, in order to generate the glueball the quarks have to be annihilated via the QCD interaction. Compared to the form factor of $B$ to an ordinary $\bar{q} q$ transition, such a contribution is suppressed by $\alpha_{s}\left(m_{b} \Lambda_{Q C D}\right)$, where the scale in $\alpha_{s}$ has been set to the typical scale in the transition. The calculation in the perturbative QCD approach shows that the $B$-to-glueball form factor is suppressed by a factor of $6-10[31,32]$.

In the following discussion, we will neglect the small contributions from the glueball content, and thus only the $\bar{n} n(\bar{s} s)$ component will contribute in $B\left(B_{s}\right)$ decays into a scalar meson plus a $J / \psi$. As an important consequence, $\mathcal{B}_{3} / \mathcal{B}_{1}=\alpha_{2}^{2}$ and $2 \mathcal{B}_{4} / \mathcal{B}_{2}=\alpha_{3}^{2}$, while $\mathcal{R}$ directly reflects the size of the glueball component. The significant deviation of $\mathcal{R}$ from 0 may be a signal for a glueball. 


\section{Implications from the present data}

In ref. [16], the Belle Collaboration reported the observation of a scalar mesonic state $f_{X}$ from the process $B_{s} \rightarrow J / \psi f_{X} \rightarrow J / \psi \pi^{+} \pi^{-}$with a significance of $4.2 \sigma$,

$$
\mathcal{B}\left(B_{s} \rightarrow J / \psi f_{X} \rightarrow J / \psi \pi^{+} \pi^{-}\right)=\left(0.34_{-0.15}^{+0.14}\right) \times 10^{-4} .
$$

The mass and width of this resonance are determined as

$$
\begin{gathered}
m_{f_{X}}=\left(1.405 \pm 0.015_{-0.007}^{+0.001}\right) \mathrm{GeV}, \\
\Gamma_{f_{X}}=\left(0.054 \pm 0.033_{-0.003}^{+0.014}\right) \mathrm{GeV} .
\end{gathered}
$$

Subsequently, the LHCb Collaboration has found a similar resonance,

$$
\begin{aligned}
m_{f_{X}} & =(1.4751 \pm 0.0063) \mathrm{GeV} \\
\Gamma_{f_{X}} & =(0.113 \pm 0.011) \mathrm{GeV}
\end{aligned}
$$

The branching ratio (BR) of $B_{s} \rightarrow J / \psi f_{X}$ is roughly $4 \%$ of the BR for $B_{s} \rightarrow J / \psi \phi$. Remembering that $\mathcal{B}\left(B_{s} \rightarrow\right.$ $J / \psi \phi)=\left(1.09_{-0.23}^{+0.28}\right) \times 10^{-3}[28]$, we note that the $\mathrm{LHCb}$ result is consistent with the Belle measurement.

The measured branching ratio of $B_{s} \rightarrow J / \psi f_{X} \rightarrow$ $J / \psi \pi^{+} \pi^{-}$is helpful in determining the mixing coefficient $\alpha_{2}$ in $f_{X}$ together with

$$
\mathcal{B}\left(B^{0} \rightarrow J / \psi K^{0}\right)=(8.71 \pm 0.32) \times 10^{-4} .
$$

Under the assumption of factorization, we extract the coefficient $\alpha_{2}$ as

$$
\begin{aligned}
\alpha_{2}= & (0.27 \pm 0.13) \times \frac{F_{1}^{B \rightarrow K}\left(m_{J / \psi}^{2}\right)}{0.53} \\
& \times \frac{1.22}{F_{1}^{B_{s} \rightarrow f_{0}(\bar{s} s)}\left(m_{J / \psi}^{2}\right)} \times \sqrt{\frac{10 \%}{\mathcal{B}\left(f_{X} \rightarrow \pi^{+} \pi^{-}\right)}},
\end{aligned}
$$

where we have used the calculation of form factors from refs. [33,32]: $F_{1}^{B \rightarrow K}\left(m_{J / \psi}^{2}\right)=0.53$ and $F_{1}^{B_{s} \rightarrow f_{0}(\bar{s} s)}\left(m_{J / \psi}^{2}\right)=1.22$, while variations of the form factors will change the extracted coefficient. The uncertainties shown in the parenthesis are from the nonfactorizable contributions and have been conservatively taken as large as $50 \%$. The decay branching fraction of $f_{X} \rightarrow \pi^{+} \pi^{-}$is an important input in the analysis and we have used $10 \%$ for illustration.

\section{Comparison with theory}

For illustration, we will consider two widely discussed mixing mechanisms of the scalar mesons and for an overview of alternative schemes see refs. [24,34-36] and many references therein. Because the decay width of the $f_{0}(1500)$ is not compatible with the ordinary $\bar{q} q$ state, it is claimed that $f_{0}(1500)$ is primarily a scalar glueball $[21,22]$, and the mixing matrix through fitting the data of two-body decays of scalar mesons is

$$
\left(\begin{array}{l}
f_{0}(1710) \\
f_{0}(1500) \\
f_{0}(1370)
\end{array}\right)=\left(\begin{array}{ccc}
0.36 & 0.93 & 0.09 \\
-0.84 & 0.35 & -0.41 \\
0.40 & -0.07 & -0.91
\end{array}\right)\left(\begin{array}{c}
G \\
\bar{s} s \\
\bar{n} n
\end{array}\right)
$$

Based on the SU(3) assumption for scalar mesons and the quenched Lattice QCD results [9], Cheng et al. [23] reanalyzed all existing experimental data and derived the mixing coefficient matrix as

$$
\left(\begin{array}{l}
f_{0}(1710) \\
f_{0}(1500) \\
f_{0}(1370)
\end{array}\right)=\left(\begin{array}{ccc}
0.93 & 0.17 & 0.32 \\
-0.03 & 0.84 & -0.54 \\
-0.36 & 0.52 & 0.78
\end{array}\right)\left(\begin{array}{c}
G \\
\bar{s} s \\
\bar{n} n
\end{array}\right) \text {. }
$$

Here, the $f_{0}(1710)$ tends to be a glueball. This is very different from the first matrix of mixing coefficients in eq. (10), while both schemes can well explain the data on the production in $J / \psi$ and decays of the $f_{0}$.

The mixing scheme of eq. (11) predicts a much larger production branching ratio for $J / \psi \rightarrow \gamma f_{0}(1710)$ than $J / \psi \rightarrow \gamma f_{0}(1500)$ and implies a relatively pure glueball around $1.7 \mathrm{GeV}$. In contrast, the mixing scheme of eq. (10) suggests that those two nearby states $f_{0}(1500)$ and $f_{0}(1710)$ both have sizeable glueball components. It can be understood that the interferences between the glueball and $\bar{q} q$ production would lead to an enhanced production rate for $J / \psi \rightarrow \gamma f_{0}(1710)$, but a suppression of $J / \psi \rightarrow \gamma f_{0}(1500)$. Such an ambiguity reflects the lack of knowledge on the glueball- $\bar{q} q$ coupling in the scalar sector. Qualitatively speaking, it is strongly model-dependent to determine the glueball component of scalar mesons in their strong productions and strong decays. In this sense, it is interesting to recognize the advantages of probing the flavor components of the scalar mesons in $B$ decays. To be more specific, in the decay of $B_{s} \rightarrow J / \psi f_{0}$ the mixing coefficients in the second column, $\alpha_{2}$ 's defined in eq. (1), will be projected out by the weak transitions. Thus, they provide a natural filter of the $\bar{s} s$ component.

Apart from the measurements on $B_{s} \rightarrow J / \psi \pi^{+} \pi^{-}$, both the LHCb and D0 Collaborations have measured the branching ratio of the process $B_{s} \rightarrow J / \psi K^{+} K^{-}[18,19]$, in which no significant evidence for any scalar resonance decaying into $K^{+} K^{-}$is found. Thus, it may be hard to interpret the $f_{0}(1710)$ as an $\bar{s} s$ state since in this case the $f_{0}(1710)$ mainly decays into $K^{+} K^{-}$. From this viewpoint, it seems that the mixing in eq. (10) is less favored compared to the one in eq. (11), where the production of the glueball dominated $f_{0}(1710)$ is expected to be suppressed.

The present experimental precision does not allow a conclusion for the $f_{0}(1500)$. Although in the mixing scheme of eq. (11), the $f_{0}(1500)$ is favored to be produced via its $\bar{s} s$ component, its decay branching ratio to $K^{+} K^{-}$ is relatively small, i.e. $(8.6 \pm 1.0) \%$ [28]. With higher statistics available in the future, a determination of the relative production rates for the $f_{0}(1710)$ and the $f_{0}(1500)$ in $B_{s} \rightarrow J / \psi \pi^{+} \pi^{-}$should be able to provide crucial information about their internal structure. 
Supposing that the absence of $f_{0}(1710)$ in $K^{+} K^{-}$is indeed due to the dominance of an internal glueball component, one notices that the such a scenario is consistent with the recent Lattice QCD calculation of ref. [37], where the $f_{0}(1710)$ as a glueball candidate would have a large production rate in $J / \psi$ radiative decays, i.e. $J / \psi \rightarrow$ $\gamma f_{0}(1710)$. It is also in support from the coupled channel study of the S-waves meson-meson scattering [38] (for different opinions see refs. $[39,40])$, in which the $f_{0}(1710)$ and a pole at $1.6 \mathrm{GeV}$, which is an important contribution to the $f_{0}(1500)$, are identified as the scalar glueballs.

Regarding the $f_{X}$ resonance discovered by Belle and $\mathrm{LHCb}$, we explore two cases since the masses and widths of both $f_{0}(1370)$ and $f_{0}(1500)$ are close to the experimental values:

i) The $f_{X}$ can be the $f_{0}(1500)$. From the PDG tables [28], we quote

$$
\mathcal{B}\left(f_{0}(1500) \rightarrow \pi^{+} \pi^{-}\right)=\frac{2}{3} \times 34.8 \%=23.2 \%,
$$

which implies

$$
\left|\alpha_{2}\right|=(0.18 \pm 0.09)
$$

Such a small value seems to favor the mixing matrix shown in eq. (10).

ii) The $f_{X}$ can be the $f_{0}(1370)$. The PDG quote that the $f_{0}(1370) \rightarrow \rho \rho$ is its main decay mode. Both WA102 [41] and BES-II [42] found that the branching ratio fraction of $f_{0}(1370) \rightarrow \pi^{+} \pi^{-}$over $f_{0}(1370) \longrightarrow$ $K^{+} K^{-}$is small, i.e. $\sim 20 \%$ [42]. If this is the case, the extracted coefficient $\alpha_{2}$ is of a similar size as the value in eq. (13). In such a situation, it seems hard to distinguish the mixings given in eqs. (10) and (11).

\section{Future improvements}

Although the present experimental status does not allow us to make a definite conclusion on the $f_{X}$, we would expect that the situation will be greatly improved in the future. As we have shown above, the measurement of branching ratios of the $B_{s} \rightarrow J / \psi f_{0}$ with high statistics will be able to pin down the flavor components of the scalars. Therefore, a precise measurement of the relative production rates of all (or some of) those scalars in $B_{s} \rightarrow J / \psi f_{0}$ will be an ideal $\bar{s} s$ filter for the determination of the $\bar{s} s$ components inside those scalar mesons. It is also possible to use the $B \rightarrow J / \psi f_{0}$ decays as a flavor filter for the non-strange $\bar{q} q$ components similar to that in $B_{s} \rightarrow J / \psi f_{0}$. A combined measurements of $B_{s} \rightarrow J / \psi f_{0}$ and $B \rightarrow J / \psi f_{0}$ will be very selective to scalar mixing models and can be compared with the scalar production mechanisms studied in, e.g., $J / \psi \rightarrow \gamma f_{0}$.
Generically the branching fractions of the $b \rightarrow \bar{c} c d$ processes are suppressed by $V_{c d}^{2} / V_{c s}^{2} \sim 0.04$, which we suppose would be compensated by the large luminosity of the future experiments. Based on the $1 \mathrm{fb}^{-1}$ of data sample collected at $7 \mathrm{TeV}$, the $\mathrm{LHCb}$ Collaboration has analyzed the resonant components in $B \rightarrow J / \psi \pi^{+} \pi^{-}$[20]. The Super KEKB factory is expected to gather about $50 \mathrm{ab}^{-1}$ of data, which is two orders of magnitude larger than the data sample collected on the KEKB collider [43]. With such a high statistic data base, one might gain access to $B_{s} / B_{d} \rightarrow J / \psi f_{0}(\gamma \gamma)$ in which the scalar meson is reconstructed in the two-photon final state. Compared to the $B_{s} / B \rightarrow J / \psi f_{0}\left(\pi^{+} \pi^{-}, K^{+} K^{-}\right)$, in which the decay of the $f_{0}$ is not under control due to the unknown contributions from the glueball, the $B_{s} / B_{d} \rightarrow J / \psi f_{0}(\gamma \gamma)$ is cleaner. Due to the fact that the gluons are free of electromagnetic interaction, the glueball component will not contribute. Thus, the decay matrix elements of the three $f_{0}$ states can be determined by the mixing coefficients and electric charges of the flavor components.

\section{Conclusion}

To summarize, using the available experimental data we have demonstrated that the flavor $\mathrm{SU}(3)$ symmetry for the light quarks holds quite well and can be applied to the study of $B$ decays into a scalar meson plus a charmonium. Our analysis suggests that such a process would serve as an ideal flavor filter for probing the quark contents of scalar mesons. In the presence of mixings between a glueball and ordinary $\bar{q} q$ mesons, we show that the mixing parameters can be extracted and explicitly related to experimental data from the LHCb, Belle and Do Collaborations. Although the present experimental data sample does not allow a solid conclusion on all those states, we have shown the sensitivities of such a probe to the scalar structures. The future Super KEKB factory would allow access to establishing the mixing pattern among those three scalars, and possibly allow the long-standing puzzle concerning the existence and mixings of the scalar glueball predicted by QCD to be disentangled.

WW thanks Y.L. Shen for useful discussions and Institute of High Energy Physics and Tianjin University for their hospitalities during his visit when part of this work was done. This work is supported in part by the DFG and the NSFC through funds provided to the Sino-German CRC 110 "Symmetries and the Emergence of Structure in QCD", the "EU I3HP Study of Strongly Interacting Matter" under the Seventh Framework Program of the EU, and the NSFC under the Grant Nos. 11228512, 11235005, 11075168, and 11035006.

Open Access This is an open access article distributed under the terms of the Creative Commons Attribution License (http://creativecommons.org/licenses/by/3.0), which permits unrestricted use, distribution, and reproduction in any medium, provided the original work is properly cited. 


\section{References}

1. UKQCD Collaboration (G.S. Bali et al.), Phys. Lett. B 309, 378 (1993).

2. H. Chen, J. Sexton, A. Vaccarino, D. Weingarten, Nucl. Phys. Proc. Suppl. 34, 357 (1994).

3. C.J. Morningstar, M.J. Peardon, Phys. Rev. D 60, 034509 (1999).

4. A. Vaccarino, D. Weingarten, Phys. Rev. D 60, 114501 (1999).

5. C. Liu, Chin. Phys. Lett. 18, 187 (2001).

6. D.Q. Liu, J.M. Wu, Y. Chen, High Energy Phys. Nucl. Phys. 26, 222 (2002).

7. N. Ishii, H. Suganuma, H. Matsufuru, Phys. Rev. D 66 , 014507 (2002).

8. M. Loan, X.Q. Luo, Z.H. Luo, Int. J. Mod. Phys. A 21, 2905 (2006).

9. Y. Chen et al., Phys. Rev. D 73, 014516 (2006).

10. X.-G. He, T.-C. Yuan, hep-ph/0612108.

11. C.-H. Chen, T.-C. Yuan, Phys. Lett. B 650, 379 (2007).

12. H.G. Dosch, S. Narison, Nucl. Phys. Proc. Suppl. 121, 114 (2003).

13. P. Minkowski, W. Ochs, Eur. Phys. J. C 39, 71 (2005).

14. G. Mennessier, S. Narison, X.-G. Wang, Phys. Lett. B 696, 40 (2011)

15. W. Ochs, J. Phys. G 40, 043001 (2013).

16. Belle Collaboration (J. Li et al.), Phys. Rev. Lett. 106, 121802 (2011).

17. LHCb Collaboration (R. Aaij et al.), Phys. Rev. D 86, 052006 (2012).

18. LHCb Collaboration (R. Aaij et al.), Phys. Rev. Lett. 108, 151801 (2012).

19. D0 Collaboration (V.M. Abazov et al.), Phys. Rev. D 86, 092011 (2012).

20. LHCb Collaboration (R. Aaij et al.), Phys. Rev. D 87, 052001 (2013).

21. F.E. Close, A. Kirk, Phys. Lett. B 483, 345 (2000).

22. F.E. Close, Q. Zhao, Phys. Rev. D 71, 094022 (2005).
23. H.Y. Cheng, C.K. Chua, K.F. Liu, Phys. Rev. D 74, 094005 (2006).

24. C. Amsler, S. Eidelman, T. Gutsche, C. Hanhart, S. Spanier, N.A. Törnqvist, Note on Scalar Mesons Below $2 G E V$, review published in [28].

25. P. Minkowski, W. Ochs, Eur. Phys. J. C 9, 283 (1999).

26. P. Colangelo, F. De Fazio, W. Wang, Phys. Rev. D 83, 094027 (2011).

27. P. Colangelo, F. De Fazio, W. Wang, Phys. Rev. D 81, 074001 (2010).

28. Particle Data Group Collaboration (J. Beringer et al.), Phys. Rev. D 86, 010001 (2012).

29. LHCb Collaboration (R. Aaij et al.), arXiv:1210.2631 [hep$\mathrm{ex}$.

30. Belle Collaboration (M.C. Chang et al.), Phys. Rev. D 85, 091102 (2012).

31. W. Wang, Y.-L. Shen, C.-D. Lu, J. Phys. G 37, 085006 (2010).

32. R.-H. Li, C.-D. Lu, W. Wang, X.-X. Wang, Phys. Rev. D 79, 014013 (2009).

33. C.D. Lu, W. Wang, Z.T. Wei, Phys. Rev. D 76, 014013 (2007).

34. C. Amsler, N.A. Tornqvist, Phys. Rep. 389, 61 (2004).

35. E. Klempt, A. Zaitsev, Phys. Rep. 454, 1 (2007).

36. M. Chanowitz, Phys. Rev. Lett. 95, 172001 (2005).

37. L.-C. Gui, Y. Chen, G. Li, C. Liu, Y.-B. Liu, J.-P. Ma, Y.-B. Yang, J.-B. Zhang, Phys. Rev. Lett. 110, 021601 (2013).

38. M. Albaladejo, J.A. Oller, Phys. Rev. Lett. 101, 252002 (2008).

39. L.S. Geng, E. Oset, Phys. Rev. D 79, 074009 (2009).

40. L.S. Geng, F.K. Guo, C. Hanhart, R. Molina, E. Oset, B.S. Zou, Eur. Phys. J. A 44, 305 (2010).

41. WA102 Collaboration (D. Barberis et al.), Phys. Lett. B 479, 59 (2000).

42. BES Collaboration (M. Ablikim et al.), Phys. Lett. B 607, 243 (2005)

43. T. Aushev, W. Bartel, A. Bondar, J. Brodzicka, T.E. Browder, P. Chang, Y. Chao, K.F. Chen et al., arXiv:1002. 5012 [hep-ex]. 